\title{
THE INCIDENCE AND CHARACTERISTIC FEATURES OF ANEMIA IN OLDER PATIENTS WITH CORONARY ARTERY DISEASE Alpha James ${ }^{1}$, Nataliia Pavliukovych, ${ }^{2}$ Oleksandr Pavliukovych ${ }^{3}$
}

\begin{abstract}
:
INTRODUCTION: Previous studies have shown that the presence of anemia is associated with increased short- and longterm outcomes in patients with cardio-vascular diseases, especially coronary artery disease. Decreased hemoglobin level is associated with increased mortality, increased frequency of hospitalization due to different reasons, decreased quality of life and worsening of the clinical course of the main and comorbid diseases. Knowledge about the origin of the patient's anemia should result in its treatment and possible prevention of the rapid progression of the main cardio-vascular disease, particularly in elderly patients.

OBJECTIVES: determination of the incidence and characteristic features of anemia in elderly and senile patients with chronic forms of coronary artery disease.

METHODS: 1993 case reports of patients with chronic forms of coronary artery disease with comorbid anemia were analyzed retrospectively.

RESULTS: Among all examined patients with coronary artery disease, anemia is found in nearly $70 \%$ of cases. In people after the age of 60, anemia is more common in men than in women. Only in less than a hundred case reports, the diagnosis of anemia was recorded in the final clinical diagnosis during patients' discharge from the hospital. The incidence of anemia does not depend on the form of the chronic CAD (stable angina pectoris or cardiosclerosis). In most patients with CAD, comorbid anemia is of a normochromic and normocytic character. Along with the progression of the severity of the comorbid anemia, a statistically significant increase of the hospitalization period is observed. In patients with CAD and comorbid anemia the frequency of hospitalizations per year is also increased.

CONCLUSIONS: Chronic forms of coronary artery disease in elderly and senile patients in $69,89 \%$ of cases are complicated by comorbid anemia of different degrees of severity. In older patients with CAD, anemic syndrome is most often caused by respiratory diseases, stomach and duodenal ulcers, and cancer of various localizations. In most patients with CAD, comorbid anemia is of a normochromic and normocytic character. Concomitant anemia in patients with CAD contributes to the prolongation of the patients' in-hospital treatment and increases the frequency of hospitalizations due to the main disease throughout the year.
\end{abstract}

UDC Classification: 616.1; DOI: http://dx.doi.org/10.12955/cbup.v6.1270

Keywords: cardio-vascular disease, coronary artery disease, anemia

Introduction. According to Kovalenko et al. (2016) cardiovascular diseases have been among the leading causes of mortality in Ukraine for many years. An important role in their occurrence belongs to the negative "achievements" of modern society: hypodynamia, increased caloric content of food products, chronic mental stress...etc. The indicated well-known factors contribute to the unceasing increase in the incidence of coronary artery disease (CAD) arterial hypertension, obesity, diabetes mellitus, dyslipidemia.

Clinical trials convincingly point to the important role of reduced hemoglobin level in the progression of cardiovascular diseases. Anemia is recognized as an independent predictor of a high risk of fatal cardiovascular events developing during 6 years in non-cardiovascular patients, especially those aged 45-64. In the PRAISE study in patients with severe chronic heart failure with decreased hematocrit to $25.4-37.4 \%$, the risk of sudden death was $52 \%$ higher than with its high value $(46.1-58.8 \%)$. In addition, the reduction of this figure by $1 \%$ below $25.4 \%$ was accompanied by an increase in the risk of mortality by $11 \%$.

Anemia is associated with increased all-cause hospitalization and mortality in community-dwelling individuals above the age of 65. It is known by Gandhi et al. (2017) that in primary care offices higher prevalence of anemia is associated with advancing age and comorbidities, such as essential hypertension, hypothyroidism, chronic kidney disease, malignancy, rheumatologic disease, congestive heart failure, and coronary artery disease. In the large population with stable CAD, according to Nappi (2003) and Kalra et al. (2017), low hemoglobin is an independent predictor of mortality, cardiovascular events, and major bleeds. Persisting or new-onset anemia is a powerful predictor of cardiovascular and non-cardiovascular mortality.

\footnotetext{
${ }^{1}$ Higher education institution in Ukraine «Bukovinian State Medical University», alphajames608@ gmail.com

${ }^{2}$ Higher education institution in Ukraine «Bukovinian State Medical University», natasha.pavlyukovich@gmail.com

${ }^{3}$ Higher education institution in Ukraine «Bukovinian State Medical University», dr.alex2@ rambler.ru
} 
Aim: to determine the incidence and characteristic features of anemic syndrome (AS) in patients with CAD of elderly and senile age.

\section{Material and Methods of Investigation}

An unicenter retrospective study of 1993 patient records of illnesses was carried out. Patients were undergoing inpatient treatment in the cardiological department of the Regional Hospital for Disabled Soldiers of the Great Patriotic War (Chernivtsi, Ukraine) from January to December 2014. All patients were diagnosed with chronic CAD, which was represented by stable angina pectoris II-III functional classes (according to the classification of the Canadian Association of Cardiologists, 1976), as well as diffuse and focal (post-infarction) cardiosclerosis. The age of the patients ranged from 60 to 96 years (average age 79.5 \pm 5.24 years). Comorbid anemia was diagnosed in case of hemoglobin level below $130 \mathrm{G} / \mathrm{l}$ in males and $120 \mathrm{G} / \mathrm{l}$ in females (WHO's Recommendations, 2003).

Distribution of patients by age is given in Figure 1. The data from 252 elderly patients (60-74 years old) $-12.64 \%$ and 1700 senile patients (75-89 years old) $-85.30 \%$ were analyzed. In addition, the investigation included records of the hospitalized patients over the age of $90(2.06 \%)$. Gender distribution of the patients is represented in Figure 2.

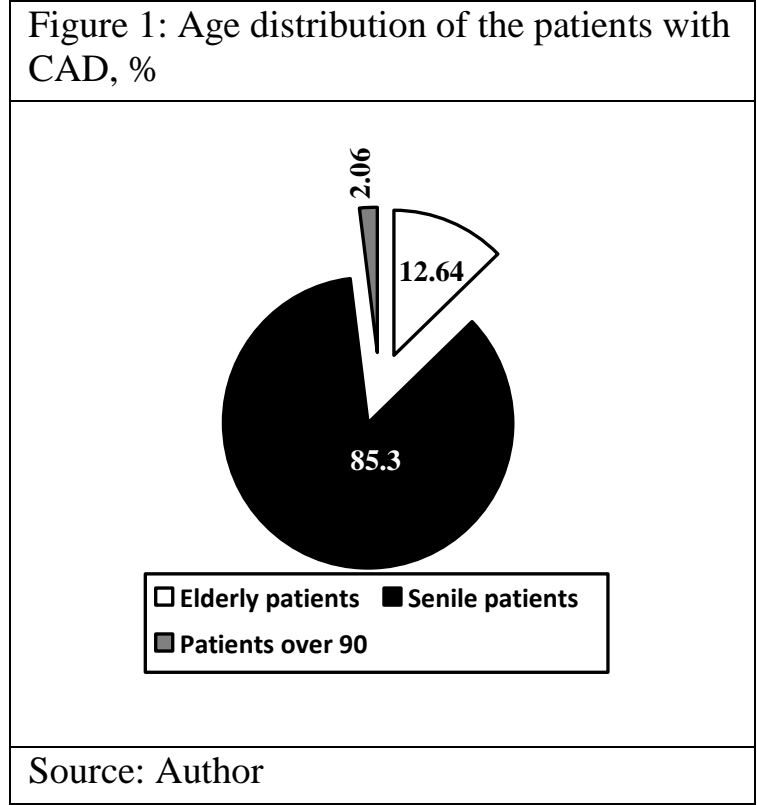

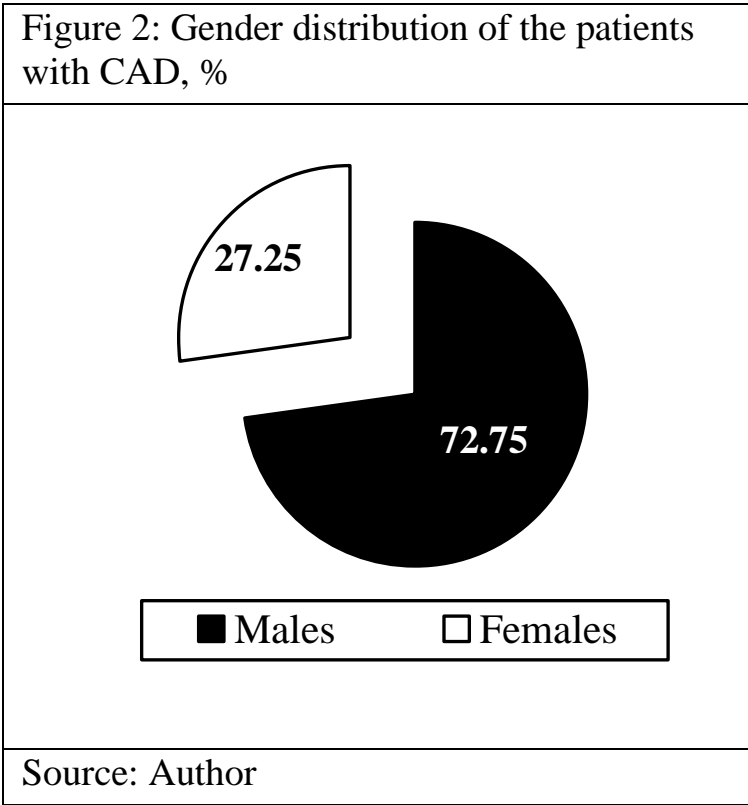

\section{Results of the Investigation}

Among all examined patients, AS was found in 69.89\% (1393 cases), which coincides with the literature data. Among male patients, anemia occurred in 1120 cases, which was $77.24 \%$; among women it occurred in 273 cases $(50.28 \%$ ). It was established that in people after the age of 60 , anemia is more common in men than in women, whereas it is a well-known fact that in adolescents and young adults AS occurs more frequently in females.

Mild anemia (hemoglobin level 90-120 (130) G/l) was the most frequent comorbid pathology in patients with CAD (1317 cases, 94.54\%). Moderate degree of anemia severity (hemoglobin level 70$90 \mathrm{G} / \mathrm{l})$ occurred in 65 cases $(4.67 \%)$. In $11 \mathrm{CAD}$ patients, severe anemia (hemoglobin level less than $70 \mathrm{G} / \mathrm{l})$ was found $(0.79 \%)$. Among males, mild anemia was detected in 1065 cases $(95.09 \%)$, moderate anemia - in 49 cases $(4.37 \%)$, severe anemia - in 6 cases $(0.54 \%)$. In women, anemia of mild degree occurred in $92.31 \%$ ( 252 cases), moderate $-5.86 \%$ ( 16 cases), severe degree $-1.83 \%$ ( 5 cases).

Only in less than a hundred case reports, the diagnosis of AS was recorded in the final clinical diagnosis during the patients' discharge from the hospital. Mild anemia was documented as a separate diagnosis in only 37 patients among $1317(2.81 \%$ ) (Figure 3). Moderate anemia was present as a separate diagnosis in about $2 / 3$ of all cases (in 42 patients out of 65 ). Approximately one-third of all cases $(36.36 \%)$ of severe anemia were not diagnosed in a hospital and no appropriate correction of hemoglobin level was performed (Figure 4). 

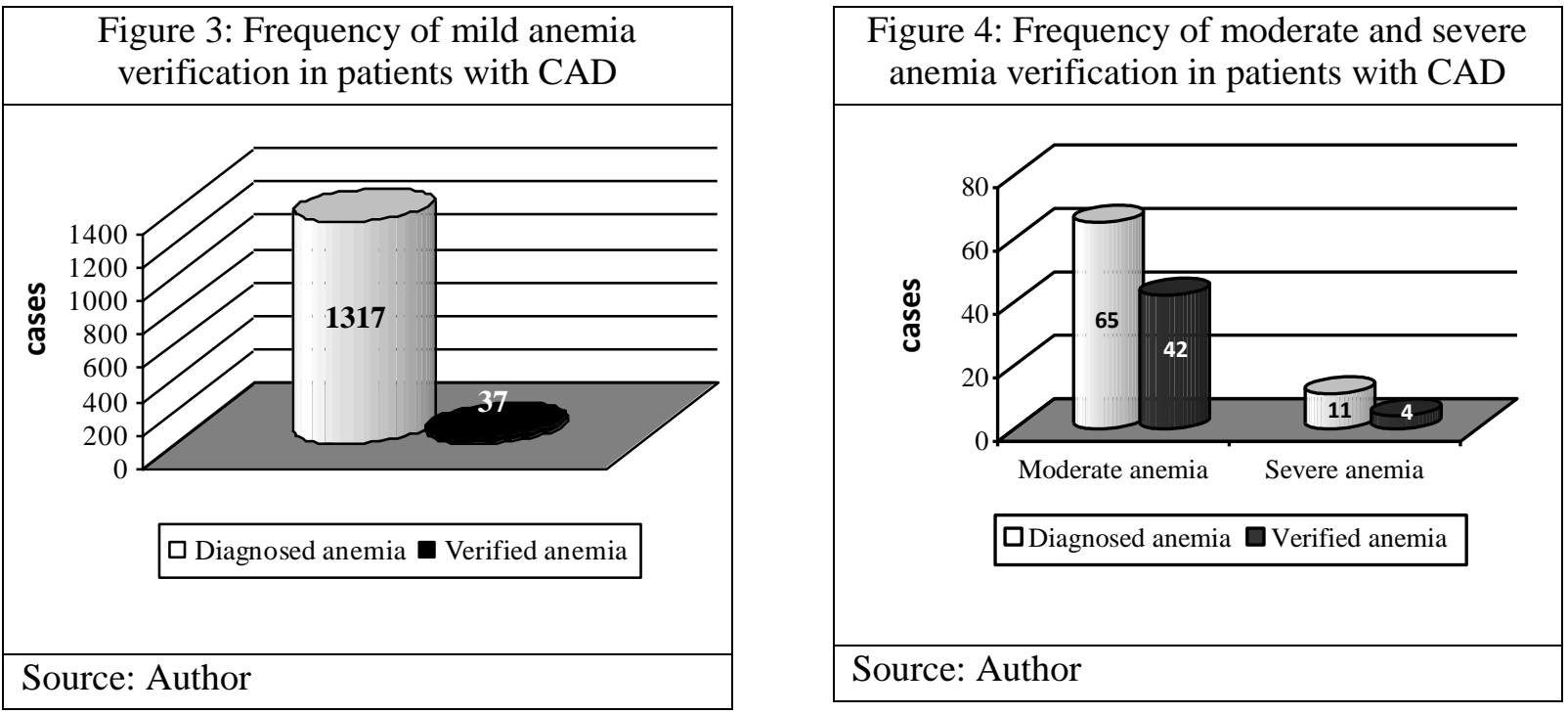

Comparing the incidence of the comorbid anemia depending on the form of chronic CAD, we have found out that anemia in approximately equal percent of cases complicated the course of stable angina pectoris and post-infarction or diffuse cardiosclerosis (Table 1).

Table 1: Dependence of the frequency of anemic syndrome on the form of chronic CAD
\begin{tabular}{|c|c|c|c|c|}
\hline Form of CAD & Stable angina pectoris & \multicolumn{2}{c|}{ Cardiosclerosis } \\
\hline Anemia & + & - & + & - \\
\hline Absolute number & 753 & 219 & 640 & 381 \\
\hline$\%$ & 77.47 & 22.53 & 62.68 & 37.32 \\
\hline
\end{tabular}

\section{Source: Author}

Comparing the degree of saturation of erythrocytes with hemoglobin, the hyperchromic character of anemia (color index>1.05) was detected in 22 patients $(1.58 \%)$, hypochromic (color index $<0.86$ ) - in 116 patients $(8.33 \%)$. In most cases anemia in patients with CAD was of normochromic character 1255 cases $(90.09 \%)$ (Figure 5$)$.
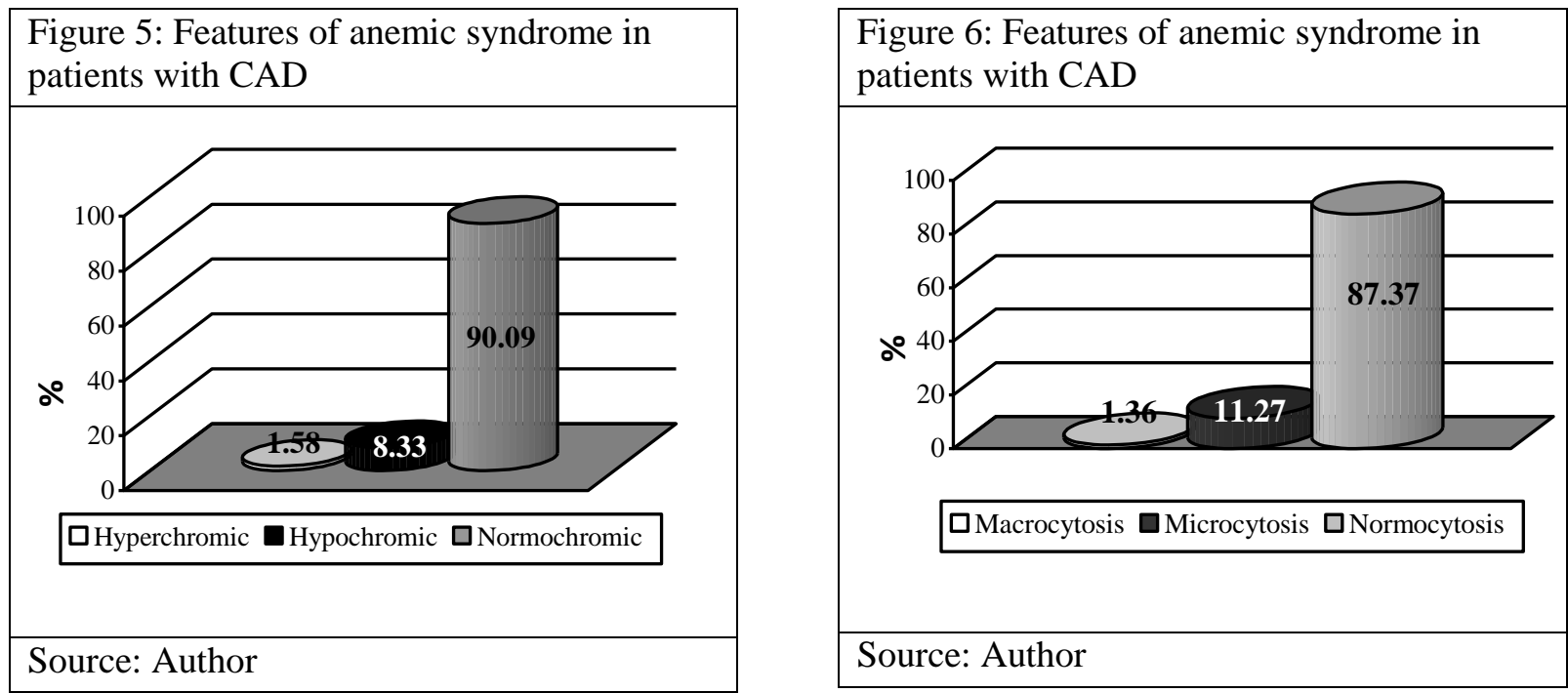

To determine the morphological characteristics of the AS in patients with chronic forms of CAD, the erythrocytes mean corpuscular volume (MCV) was calculated. Mild macrocytosis (MCV=95-108 fl) 
was detected only in several cases - 19 patients, $(1.36 \%)$, microcytosis $(\mathrm{MCV}<80 \mathrm{fl})$ - in 157 patients (11.27\%), normocytosis was found in 1217 cases $(87.37 \%$ ) (Figure 6).

Thus, in most patients with CAD, comorbid anemia is of a normochromic and normocytic character.

The average duration of the in-hospital treatment of the patients with CAD without anemia was $16.14 \pm 0.03$ days. Along with the progression of the severity of the comorbid anemia, a statistically significant increase of the hospitalization period was observed. So, if CAD was accompanied by mild anemia, the average in-hospital treatment duration was $18.23 \pm 0.050$ days, moderate anemia $20.02 \pm 0.077$, severe anemia $-21.03 \pm 0.100$. In patients with CAD and comorbid anemia, the frequency of hospitalizations per year was also increased. Thus, among all patients with CAD without anemia, $85.50 \%$ of the patients were hospitalized twice a year due to the main disease, $43.01 \%$ - three times per year, $24.33 \%$ - four times per year, $0.33 \%$ - more than four times. On the other hand, in the case of CAD with concomitant anemia, $94.54 \%$ of patients were admitted to the hospital twice a year, $55.20 \%$ - three times, $33.17 \%$ - four times, $3.88 \%$ - more often.

Among all analyzed cases, in the largest number of them (79.83\%) CAD and anemia were comorbid with chronic obstructive pulmonary disease, $28.14 \%$ of patients suffered from peptic ulcers of the stomach and duodenum, $4.31 \%$ of patients had oncological pathology of various localization, and in $20.32 \%$ of cases anemia and CAD were isolated.

\section{Conclusion}

Chronic forms of coronary artery disease in elderly and senile patients are complicated by comorbid anemia of different degrees of severity in $69.89 \%$ of cases, which correlates with the data obtained by Chonchol M., Nielson C. (2008), Grammer T.B., Kleber M.E., Silbernagel G., Pilz S. (2014), Silva C.L., Lima-Costa M.F., Firmo J.O., Peixoto S.V. (2013). This fact shows an increased risk of death and major clinical adverse events among elderly patients with stable symptomatic CAD (Muzzarelli S., Pfisterer M. (2006). In older patients with CAD, anemic syndrome is most often caused by respiratory diseases $(79.83 \%)$, stomach and duodenal ulcers $(28.14 \%)$, and cancer of various localizations $(4.31 \%)$. Concomitant anemia in patients with CAD contributes to the prolongation of the patients' in-hospital treatment and increases of the frequency of hospitalizations due to the main disease throughout the year.

\section{References}

Kovalenko V.M., Lutay M.I., Sirenko Yu. M., Sychov O.S. (2016) Cardio-vascular diseases. Classification, standards of diagnosis and treatment. 189. (in Ukrainian)

Gandhi S.J., Hagans I., Nathan K., Hunter K., Roy S. (2017) Prevalence, comorbidity and investigation of anemia in the primary care office. J. Clin. Med. Res. 2017.9 (12). 970-980.

Kalra P.R., Greenlaw N., Ferrari R., Ford I. et al. (2017) Hemoglobin and change in hemoglobin status predict mortality, cardiovascular events, and bleeding in stable coronary artery disease. Am. J. Med. 130 (6). 720-730.

Nappi J. (2003) Anemia in patients with coronary artery disease. Am. J. Health Syst. Pharm. 60 (14 Suppl 3). S4-8.

Muzzarelli S., Pfisterer M. (2006) Anemia as independent predictor of major events in elderly patients with chronic angina. Am Heart J. 152(5). 991-996.

Chonchol M., Nielson C. (2008).Hemoglobin levels and coronary artery disease. Am Heart J. 155(3). 494-498.

Grammer T.B., Kleber M.E., Silbernagel G., Pilz S. (2014) Hemoglobin, iron metabolism and angiographic coronary artery disease (The Ludwigshafen Risk and Cardiovascular Health Study). Atherosclerosis. 2014. 236(2). 292-300.

Silva C.L., Lima-Costa M.F., Firmo J.O., Peixoto S.V. (2013) Anemia and hemoglobin level as prognostic factors of mortality in community-dwelling elderly: evidence from the Bambuí Cohort Study on Aging, Minas Gerais State, Brazil. Cad Saude Publica. 2013. 29(11). 2241-2250. 